\title{
Padrões de Projetos para Organizações de Contratos Inteligentes
}

\author{
Alan Veloso ${ }^{1,2}$, Leonardo da Costa ${ }^{1}$, Billy Pinheiro ${ }^{1}$, \\ Antônio Abelém ${ }^{1}$, Filipe Saraiva ${ }^{2}$ \\ ${ }^{1}$ Grupo de Redes de Computadores e Comunicação Multimídia (GERCOM) \\ ${ }^{2}$ Laboratório de Inteligência Artificial Aplicada (LAAI) \\ Universidade Federal do Pará (UFPA) - Belém - Pará - Brasil \\ alan. veloso@icen.ufpa.com, \\ $\{l b c, b i l l y$, abelem, saraiva\}@ufpa.br
}

\begin{abstract}
Currently, different areas are using blockchain and smart contracts to provide security to systems. These systems can use multiple smart contracts that coordinate and cooperate with each other to achieve their goals, that is, they form an smart contracts organization (SCO). This study preliminarily presents design patterns from different organizational structures based on the literature on Multiagent System (MAS) and SCO, which include hierarchy, holarchy, society and market. The design patterns are briefly described, the systems that present them are exposed and a form of evaluation and validation is proposed.
\end{abstract}

Resumo. Atualmente diferentes áreas estão utilizando blockchain e contratos inteligentes para prover segurança aos sistemas. Esses sistemas podem utilizar múltiplos contratos inteligentes que se coordenam e cooperam entre si para alcançar seus objetivos, ou seja, formam uma organização de contratos inteligentes (Smart Contracts Organization - SCO). Este estudo apresenta preliminarmente padrões de projetos de diferentes estruturas organizacionais baseadas na literatura de Sistema Multiagente (Multiagent System - MAS) e na de $S C O$, que incluem hierarquia, holarquia, sociedade e mercado. Os padrões de projetos são descritos de maneira sucinta, os sistemas que os apresentam são expostos e uma forma de avaliação e validação é proposta.

\section{Introdução}

Atualmente, várias áreas utilizam a tecnologia blockchain e contratos inteligentes para prover segurança a sistemas, como cidades inteligentes, e-saúde, industria 4.0 e outros [Casino et al. 2019]. Entretanto, um problema ainda existente é carência de soluções para auxiliar o desenvolvimento de Organizações de Contratos Inteligentes (Smart Contracts Organzation - SCO), ou seja, coordenação e cooperação de mais de um contrato inteligente.

Assim, decorrente da atual similaridade entre contratos inteligentes e agentes reativos, este estudo fundamenta-se na literatura de Sistema Multiagente (Multiagent System - MAS) [Wooldridge 2009] para contribuir com o estado da arte sobre SCO. Desse modo, os contratos são os agentes e a blockchain é o ambiente. Seguindo essa correlação, a SCO 
é composta de contratos que coordenam suas operações para contribuir em atingir o objetivo da organização [Puranam et al. 2014], ou seja, os objetivos individuais dos contratos serão direcionados para alcançar o objetivo global.

Um aspecto que está presente na constituição de organizações inteligentes de contrato e que contribui para sua eficácia é a estrutura organizacional. Esse aspecto pode ser utilizado para limitar as interações, aumentar a redundância do sistema, formalizar objetivos de alto nível ou impor mecanismos de coordenação para garantir a execução de tarefas. Apesar dessas possibilidade de utilização, a literatura da área atualmente ainda carece de estudos que se preocupam com a estrutura organizacional em SCO, eles são direcionados a estudos de aspectos de individuais de contratos inteligentes [Bartoletti and Pompianu 2017, Worley and Skjellum 2019].

Diante disso, o presente estudo, ainda em andamento, apresenta sucintamente padrões de projetos de estruturas organizacionais em SCO, fornecendo aos profissionais interessados o conjunto de possibilidades, seus objetivos, aplicabilidades e exemplos de sistemas que utilizam a SCO, que podem ser usados como base para o desenvolvimento de novas aplicações.

Apesar de fornecer os padrões de projetos, ainda é necessário realizar uma avaliação do desempenho de cada uma das estruturas organizacionais para melhor determinar a eficiência de cada uma e uma validação da reutilização dos padrões de projetos. Nesse intuito, este estudo utilizará métricas definidas pela ISO/IEC 25023:2016 [International Organization for Standardization 2016].

O artigo está organizado da seguinte forma: a Seção 2 apresenta sucintamente a metodologia utilizada para o desenvolvimento do estudo. Posteriormente, a Seção 3 apresenta os padrões de projetos e também uma análise a respeito dos resultados obtidos. Por fim, a Seção 4 faz considerações a respeito do trabalho e possíveis próximos passos.

\section{Metodologia}

A metodologia utilizada no presente estudo foi dividida em três partes. Na primeira, foram identificadas as principais estruturas organizacionais em MAS. Na segunda, um estudo bibliográfico e documental foi realizado para identificar sistemas que utilizam SCO. $\mathrm{Na}$ última parte, foi realizado um estudo empírico para identificar quais as estruturas organizacionais encontradas em MAS estão presentes nas SCO e como são formados nelas.

O levantamento dos sistemas que utilizam SCO tratou primeiramente de uma revisão sistemática nas bases de dados, Scopus, Compendex, IEEE Xplore e ACM Digital Library para encontrar estudos de aplicações de SCO. Grande parte dos estudos encontrados foram descartado pelo critério de exclusão: Ser uma organização de contratos, ou seja, mais de um contrato. Portanto, foi necessário expandir a busca, assim, uma pesquisa informal foi realizada para coletar sistemas que usam SCO conhecidas na área e por profissionais do campo.

Por fim, a análise dos contratos inteligentes das SCO foi feita para poder identificar a estrutura organizacional. Para isso, foi necessário diferenciar em contratos que desempenham funções e contratos que são interfaces para entes internos (tokens). Assim, com o conhecimento prévio das estruturas organizacionais de MAS, foi possível determinar a estrutura organizacional que os contratos inteligentes das SCO assumem. 


\section{Resultados}

Para a identificação das estruturas organizacionais nas SCO utilizou-se o trabalho de Horling e Lesser [Horling and Lesser 2004], referência base no campo de estudo em SMA. Nas subseções a seguir descreve-se o objetivo e as aplicabilidades de cada uma das estruturas, além de sua formação em três sistemas que utilizam SCO (The DAO, Bitnation e Ethereum), que representam a parte significativa do conjunto de estruturas levantadas. Como critério de seleção destes sistemas, se considerou a notoriedade dos mesmo no âmbito de blockchain.

\subsection{Hierarquia}

$\mathrm{Na}$ estrutura, os contratos inteligentes são dispostos em forma de árvore, onde o fluxo de dados flui de contratos inteligentes de níveis inferiores para os níveis superiores, fornecendo uma visão geral para estes últimos, enquanto o fluxo de controle flui no sentido oposto à medida que contratos inteligentes de níveis superiores fornecem orientação para os que estão abaixo. Hierarquias são utilizadas quando um contrato inteligentes quer decompor uma tarefa para ser executada por outros contratos ou quando um contrato quer reduzir a carga local delegando para outros uma tarefa.

\subsubsection{Exemplo}

The DAO [Jentzsch 2016] é o sistema de maior notoriedade na literaturade blockchain. Têm por objetivo a intermediação de financiamentos coletivos, se assimilando ao Kickstarter ${ }^{1}$.

A proposta aprovada para ser financiada é desenvolvida por meio de um contrato inteligente e parametrizada para atender aos comandos do contrato da The DAO, formando assim um hierarquia. Como poderia haver mais de uma proposta aprovada, portanto, haveria mais de um contrato recebendo influencia do contrato da The DAO. Os contratos das proposta também poderiam contratar outros contratos para desenvolver a proposta aumentando assim o nível da hierarquia.

\subsection{Holarquia}

Em holarquias, os contratos inteligentes são agrupados em grupos parcialmente autônomos denominado hólons para onde são transmitidos recursos em vez de contratos inteligentes individuais. Cada hólon é composto de uma ou mais entidades subordinadas e pode ser membro de um, ou mais hólons superordenados. A estrutura de holarquia é utilizado quando se quer decompor um tarefa que não é capaz de ser finalizada por um único agente; se quer fazer a alocação de tarefas mais eficiente; precisa resolver tarefas com requisitos maiores do que qualquer agente único pode oferecer; é mais facilmente aplicadas a domínios em que as metas podem ser recursivamente decompostas em sub tarefas que podem ser atribuídas a hólons individuais, embora isso não seja essencial.

\subsubsection{Exemplo}

A Bitnation forma essa estrutura dinamicamente. Inicialmente, é criado o hólon gênesis e definidos os membros que irão compô-lo, contratos inteligentes ou indivíduos, que formam a gerencia estratégica da Bitnation. Os membros são selecionados com base no compromisso com a Bitnation, experiência em áreas designadas de trabalho e desempe-

\footnotetext{
${ }^{1}$ https: / / www.kickstarter.com/
} 
nho ao longo do tempo. Posteriormente, para que fins operacionais específicos sejam atendidos, outros hólons devem ser formados, como exemplo a publicidade. Mas outros hólons podem ser constituídos, mesmo que não objetivam a operação da Bitnation, como a organização de eventos.

Todos os membros têm um campo de trabalho (hólon), que permite a eles maior autoridade em decisões referentes ao seu âmbito. Atualmente, a Bitnation possui diversos membros, cada um deles detém participação específica em seu hólon, que pode se referir a orçamentos, elaboração ou revisão de propostas, assim, cada holón opera com um centro de custo próprio com maior autonomia.

\subsection{Mercado}

Em mercados, os contratos inteligentes são divididos em dois grupos, compradores e vendedores. Os compradores competem fazendo lances por um conjunto comum de itens, como recursos, tarefas, serviços ou mercadorias compartilhados. Os vendedores fornecem itens para serem vendidos. Os vendedores também podem designar terceiros, chamados leiloeiros, para serem responsáveis pelo processamento de lances e pela determinação do vencedor. A estrutura de mercado é utilizado quando quer transferir um produto (e.g., físico, intelectual) em troca de algum pagamento ou quer realizar planejamento descentralizado.

\subsubsection{Exemplo}

The DAO apresenta a estrutura do mercado em dois momentos distintos: na fase inicial de operação, apresentando a definição de mercado descrita na seção 3.3; e na fase de recebimento de propostas, operando como mercado reverso ou licitação, em que o vendedor começa a receber ofertas de produtos dos compradores [Horling and Lesser 2004].

The DAO funciona como um mercado aberto para levantar capital para financiar projetos. O contrato é o vendedor e oferece tokens como um produto. Esses tokens garantem vantagens aos seus titulares, como a possibilidade de votar nas propostas recebidas pela The DAO. Como existe um limite predefinido de tokens, os compradores competirão por eles. A coordenação de compras de tokens é realizada pelo contrato da The DAO, associando internamente o tokens às contas dos compradores.

The DAO também funciona como um mercado reverso para receber a oferta do projeto. The DAO recebeu propostas de projetos de todos os interessados. Assim, existe uma concorrência para os licitantes terem suas propostas aprovadas, enquanto a avaliação das propostas foi feita pelos detentores de tokens.

\subsection{Sociedade}

A sociedade estabelece um ambiente duradouro inerentemente aberto através do qual os contratos inteligentes de diferentes capacidades podem existir e interagir. Dentro da sociedade, os contratos inteligentes podem se organizar ou ser completamente não relacionados. Aplica-se a estrutura organizacional de sociedade quando quer facilitar a construção de sistemas de contratos inteligentes abertos em larga escala ou quer disponibilizar diretrizes (leis, normas ou convenções sociais) para todos os contratos inteligentes. 


\subsubsection{Exemplo}

Blockchains não permissionadas que permitem o envio de contratos inteligentes assumem a estrutura de sociedade, como é o caso do Ethereum. O Ethereum é a primeira blockchain a permitir que qualquer indivíduo envie contratos inteligentes para a rede [Wood 2014]. Assim, como precursor desse mecanismo, o Ethereum tornou-se popular para o desenvolvimento de contratos inteligentes.

O Ethereum também permitem a existência de contratos inteligentes individuais e de organizações. Ele define a moeda usada para fazer transações, o modelo de identificação das contas dos contratos inteligentes, como fazer transações e comunicação entre contratos, além de outros aspectos.

\subsection{Análise dos Resultados}

É possível observar nas subseções anteriores que diferentes estruturas organizacionais podem ser adotadas em diferentes situações, pois algumas abordagens são mais adequadas que outras para determinadas situações [Horling and Lesser 2004]. Logo, a escolha da(s) estrutura(s) adotada(s) por um projetista deve ser direcionada pelos requisitos especificados para o sistema em questão.

Estruturas organizacionais são importantes para o desenvolvimento do SCO, mesmo implicitamente [Horling and Lesser 2004]. Corkill e Lander [Corkill and Lander 1998] citam outros fatores que justificam a necessidade de evidenciar a estrutura organizacional, incluindo recursos limitados, potencial de colaboração e redundância de trabalho. Portanto, apresentar o conjunto de possibilidades para os projetistas de SCO é necessária para torná-las mais eficientes. Ao conhecer e entender as estruturas organizacionais espera-se que o uso de uma estrutura organizacional explícita seja incentivado e facilitado.

Outras estruturas apresentadas no estudo de Horling e Lesser [Horling and Lesser 2004], mas não verificadas nas SCO analisadas nesse artigo são: coalizão, time, congregação, federação e matriz. Apesar de não terem sido identificadas, ainda há a possibilidade de serem utilizadas para desenvolver novas SCO.

\section{Considerações Finais e Orientações Futuras}

A ideia de que a estrutura organizacional faz parte do desenvolvimento de organizações de contratos inteligentes, mesmo que implicitamente, foi utilizada para o desenvolvimento desse estudo. Assim, os padrões de projetos foram idealizados de forma a auxiliar o desenvolvimento de aplicações que necessitem da coordenação de funções de contratos inteligentes. Várias áreas que utilizam blockchain e contratos inteligentes podem se beneficiar dessa proposta.

O próximo passo deste projeto, com todos os aspectos teóricos definidos, é prosseguir com uma validação experimental. Posteriormente, é necessário também realizar a avaliação de desempenho de cada estrutura organizacional. As métricas foram definidas seguindo as orientações da ISO/IEC 25023:2016 [International Organization for Standardization 2016].

Apesar de dados fornecidos pela literatura de MAS sobre o comportamento de cada uma das estruturas organizacionais, é necessário avaliar o desempenho de cada uma 
no contexto de blockchain, pois se trata de um ambiente com suas particularidades associadas. Portanto, é necessário avaliar principalmente métricas de desempenho (comportamento ao longo do tempo, utilização de recurso e capacidade de resposta) das estruturas organizacionais tanto em sistemas estáticos, no qual o projetista define os participantes, mas principalmente em formações dinâmicas, onde não se sabe previamente a quantidade de membros da organização.

Além disso, como se trata de padrões de projetos com o objetivo de auxiliar o processo de devolvimento, é necessário também valiar medidas de manutenção, como modularidade, reusabilidade, analisabilidade, modificabilidade e testabilidade.

\section{Agradecimentos}

O presente trabalho foi realizado com apoio da Coordenação de Aperfeiçoamento de Pessoal de Nível Superior - Brasil (CAPES) - Código de Financiamento 001. Os autores agradecem o apoio da Universidade Federal do Pará (UFPA).

\section{Referências}

[Bartoletti and Pompianu 2017] Bartoletti, M. and Pompianu, L. (2017). An empirical analysis of smart contracts: platforms, applications, and design patterns. In International conference on financial cryptography and data security, pages 494-509. Springer.

[Casino et al. 2019] Casino, F., Dasaklis, T. K., and Patsakis, C. (2019). A systematic literature review of blockchain-based applications: Current status, classification and open issues. Telematics and Informatics, 36(May 2018):55-81.

[Corkill and Lander 1998] Corkill, D. D. and Lander, S. E. (1998). Diversity in agent organizations. Object Magazine, 8(4):41-47.

[Horling and Lesser 2004] Horling, B. and Lesser, V. (2004). A survey of multi-agent organizational paradigms. Knowledge Engineering Review, 19(4):281-316.

[International Organization for Standardization 2016] International Organization for Standardization (2016). Systems and Software Engineering: Systems and Software Quality Requirements and Evaluation (SQuaRE): Measurement of System and Software Product Quality, volume 5. ISO.

[Jentzsch 2016] Jentzsch, C. (2016). Decentralized autonomous organization to automate governance. Technical report.

[Puranam et al. 2014] Puranam, P., Alexy, O., and Reitzig, M. (2014). What's "New" About New Forms of Organizing? Academy of Management Review, 39(2):162-180.

[Wood 2014] Wood, G. (2014). Ethereum: a secure decentralised generalised transaction ledger. Ethereum Project Yellow Paper, pages 1-32.

[Wooldridge 2009] Wooldridge, M. (2009). An Introduction to MultiAgent Systems, volume 8953 of Lecture Notes in Computer Science. John Wiley \& Sons.

[Worley and Skjellum 2019] Worley, C. R. and Skjellum, A. (2019). Opportunities, Challenges, and Future Extensions for Smart-Contract Design Patterns. In Lecture Notes in Business Information Processing, volume 57, pages 264-276. Springer International Publishing. 